\title{
Optimal Control and Vanishing Viscosity for the Burgers Equation
}

\author{
C. Castro ${ }^{1}$ F. Palacios ${ }^{2}$ and E. Zuazua ${ }^{3}$ \\ 1 Universidad Politécnica de Madrid, Spain; carlos.castro@upm.es \\ 2 Universidad Politécnica de Madrid, Spain; fpalacios@gmail.com \\ 3 Basque Center for Applied Mathematics, Bilbao, Spain; zuazua@bcamath.org
}

\subsection{Introduction}

We revisit an optimization strategy recently introduced by the authors to compute numerical approximations of minimizers for optimal control problems governed by scalar conservation laws in the presence of shocks. We focus on the one-dimensional (1-D) Burgers equation. This new descent strategy, called the alternating descent method, in the inviscid case, distinguishes and alternates descent directions that move the shock and those that perturb the profile of the solution away from it. In this chapter we analyze the optimization problem for the viscous version of the Burgers equation. We show that optimal controls of the viscous equation converge to those of the inviscid one as the viscosity parameter tends to zero and discuss how the alternating descent method can be adapted to this viscous frame.

Optimal control for hyperbolic conservation laws is a difficult topic which requires a considerable analytical effort and is computationally expensive in practice. In the last years a number of methods have been proposed to reduce the computational cost and to render this type of problem affordable.

In particular, recently, the authors have introduced the alternating descent method, which takes into account possible shock discontinuities. This chapter is devoted to revisit this method in the context of the viscous Burgers equation.

We focus on the 1-D Burgers equation although most of our results extend to more general equations with convex fluxes. Most of the ideas we develop here, although they need further developments at a technical level, apply to multi-dimensional scenarios, too.

To be more precise, given a finite time horizon $T>0$, we consider the following inviscid Burgers equation:

$$
\left\{\begin{array}{l}
\partial_{t} u+\partial_{x}\left(\frac{u^{2}}{2}\right)=0, \quad \text { in } \mathbb{R} \times(0, T), \\
u(x, 0)=u^{0}(x), \quad x \in \mathbb{R} .
\end{array}\right.
$$

We also consider its viscous counterpart 


$$
\left\{\begin{array}{l}
\partial_{t} u-\nu u_{x x}+\partial_{x}\left(\frac{u^{2}}{2}\right)=0, \quad \text { in } \mathbb{R} \times(0, T), \\
u(x, 0)=u^{0}(x), \quad x \in \mathbb{R},
\end{array}\right.
$$

where $\nu>0$.

Given a target $u^{d} \in L^{2}(\mathbb{R})$ we consider the cost functional to be minimized $J: L^{1}(\mathbb{R}) \rightarrow \mathbb{R}$, defined by

$$
J\left(u^{0}\right)=\int_{\mathbb{R}}\left|u(x, T)-u^{d}(x)\right|^{2} d x,
$$

where $u(x, t)$ is the unique entropy solution of (7.1) in the inviscid case or the unique weak solution of the viscous model (7.2). Sometimes, to make the dependence on the viscosity parameter $\nu$ more explicit, the functional $J$ will be denoted by $J_{\nu}$, although its definition is the same as that of $J$, but rather for the solutions $u_{\nu}$ of (7.2) instead of (7.1). Note that the functional above is well defined in both cases, inviscid and viscous, because of the effect on the gain of integrability of both equations: When the initial data belongs to $L^{1}(\mathbb{R})$, the solutions of both (7.1) and (7.2), for any positive time $t>0$, belong to $L^{1}(\mathbb{R}) \cap L^{\infty}(\mathbb{R})$.

Although this chapter is devoted to this particular choice of $J$, most of our analysis can be adapted to many other functionals and control problems (we refer for instance to [JaSe99] and [CaZu08], where the control variable is the nonlinearity of the scalar conservation law).

We also introduce the set of admissible initial data $\mathcal{U}_{a d} \subset L^{1}(\mathbb{R})$, that we define later in order to guarantee the existence of minimizers for the following optimization problem: Find $u^{0, \min } \in \mathcal{U}_{a d}$ such that

$$
J\left(u^{0, \min }\right)=\min _{u^{0} \in \mathcal{U}_{a d}} J\left(u^{0}\right) .
$$

Similarly, we consider the same minimization problem for the viscous model (7.2): Find $u_{\nu}^{0, \min } \in \mathcal{U}_{a d}$ such that

$$
J_{\nu}\left(u_{\nu}^{0, \min }\right)=\min _{u^{0} \in \mathcal{U}_{a d}} J_{\nu}\left(u^{0}\right) .
$$

This is one of the model optimization problems that is often addressed in the context of optimal aerodynamic design, the inverse design problem (see, for example, [GiPi01]).

As we will see, the existence of minimizers for both models, the inviscid and the viscous one, is easily established under some natural assumptions on the class of admissible data $\mathcal{U}_{a d}$ using well-known well-posedness and compactness properties of the Burgers equation. However, uniqueness is false, in general.

The first result of this chapter is a $\Gamma$-convergence result guaranteeing that any sequence of minimizers $\left\{u_{\nu}^{0, \min }\right\}_{\nu>0}$, as $\nu \rightarrow 0$, has a minimizer $u^{0, \text { min }}$ of $J$ as an accumulation point.

Obviously, when $\nu>0$, which is the common situation in practice, solutions are smooth; therefore, the alternating descent method, based on the fact 
that solutions have shock discontinuities, cannot be applied as such. But for $\nu$ small enough, solutions present quasi-shock configurations. It is therefore natural to analyze how the method can be adapted to this situation to take advantage of the presence of these quasi-shocks.

The closely related issue of numerical approximations in the inviscid case has already been discussed in [CaPa08]. Indeed, in practical applications and in order to perform numerical computations and simulations, one has to replace the continuous optimization problems above by discrete ones. In particular, in what concerns the inviscid model (7.1), it is natural to consider a discretization of system (7.1) and the functional $J$. If this is done in an appropriate way, the discrete optimization problem has minimizers that are often taken, for small enough mesh sizes, as approximations of the continuous minimizers. This convergence result was proved in [CaPa08] in a suitable class of monotone finite difference schemes, satisfying the one-sided Lipschitz condition (OSLC). These schemes introduce artificial numerical viscosity. But the analysis in [CaPa08] showed that if, in the optimization process, the fact that discrete solutions may be close to shock configurations is not used, the discrete gradient algorithm shows a very slow convergence rate. Accordingly, the method proposed in [CaPa08] combines the discrete optimization approach and continuous shock analysis to derive the alternating descent method, which performs better. It is therefore natural to address the optimal control of the viscous model (7.2) similarly by viewing it as an approximation of the inviscid one (7.1) as $\nu \rightarrow 0$ and trying to take advantage of the quasi-shock configurations when they arise. This is precisely the goal of this chapter.

Our first result guarantees the convergence of the minimizers, based on the fact that the OSLC is satisfied uniformly with respect to the vanishing viscosity parameter, which ensures compactness.

The rest of this chapter is organized as follow. In Section 7.2 we recall the basic results in [CaPa08] on the existence of minimizers for the continuous problem (7.4). In Section 7.3 we analyze the convergence of the viscous minimizers as $\nu \rightarrow 0$. In Section 7.4 we recall some known results on the sensitivity of the continuous functional by linearizing system (7.1) in the presence of a shock. In Section 7.5 we briefly recall the alternating descent method. In Section 7.6 we develop an adaptation of the method of alternating descent directions to the viscous case. In Section 7.7 we present some numerical experiments that show the efficiency of the method we have developed.

\subsection{Existence of Minimizers}

In this section we prove that, under certain conditions on the set of admissible initial data $\mathcal{U}_{a d}$, there exists at least one minimizer of $J$ and $J_{\nu}$ for all $\nu>0$.

To simplify the presentation, we consider the class of admissible initial data $\mathcal{U}_{a d}$ :

$$
\mathcal{U}_{a d}=\left\{f \in L^{\infty}(\mathbb{R}), \operatorname{supp}(f) \subset K,\|f\|_{\infty} \leq C\right\},
$$


where $K \subset \mathbb{R}$ is a bounded interval and $C>0$ a constant. Obviously, $\mathcal{U}_{a d}$ as above is a bounded set of $L^{1}(\mathbb{R})$.

The analysis we shall develop here can be extended to a much wider class of admissible sets.

Theorem 1. Assume that $\mathcal{U}_{a d}$ is as above and $u^{d} \in L^{2}(\mathbb{R})$. Then the minimization problems (7.4) and (7.5) have at least one minimizer $u^{0, \min } \in \mathcal{U}_{a d}$.

Proof. The proof is simpler when $\nu>0$ because of the regularizing effect of the viscous Burgers equation. But, in order to develop arguments that are uniform on the viscosity parameter $\nu$, it is better to give a proof for the inviscid case, which applies in the viscous one as well. Thus, in what follows, we refer to the functional $J$ although the same arguments apply for $J_{\nu}$ too.

Let $u_{n}^{0} \in \mathcal{U}_{a d}$ be a minimizing sequence of $J$. Then, by definition of $\mathcal{U}_{a d}$, $u_{n}^{0}$ is bounded in $L^{\infty}$ and there exists a subsequence, still denoted by $u_{n}^{0}$, such that $u_{n}^{0} \rightarrow u_{*}^{0}$ weakly-* in $L^{\infty}$. Moreover, $u_{*}^{0} \in \mathcal{U}_{a d}$.

Let $u_{n}(x, t)$ and $u_{*}(x, t)$ be the entropy solutions of (7.1) with initial data $u_{n}^{0}$ and $u_{*}^{0}$, respectively, and assume for the moment that

$$
u_{n}(\cdot, T) \rightarrow u_{*}(\cdot, T), \quad \text { in } L^{2}(\mathbb{R}) .
$$

Then, clearly,

$$
\inf _{u^{0} \in \mathcal{U}_{a d}} J\left(u^{0}\right)=\lim _{n \rightarrow \infty} J\left(u_{n}^{0}\right)=J\left(u_{*}^{0}\right),
$$

and we deduce that $u_{*}^{0}$ is a minimizer of $J$.

Thus, the key point is to prove the strong convergence result (7.6). Two main steps are necessary to do it. a) The relative compactness of $u_{n}(\cdot, T)$ in $L^{2}$. Taking the structure of $\mathcal{U}_{a d}$ into account and using the maximum principle and the finite velocity of propagation that entropy solutions fulfill, it is easy to see that the support of all solutions at time $t=T$ is uniformly included in the same compact set of $\mathbb{R}$. Thus, it is sufficient to prove compactness in $L_{l o c}^{2}$. This is obtained from Oleinik's one-sided Lipschitz condition

$$
\frac{u(x, t)-u(y, t)}{x-y} \leq \frac{1}{t}
$$

which guarantees in fact a uniform bound of the $B V$-norm of $u_{n}(\cdot, T)$, locally in space (see $[\mathrm{BrOs} 88]$ ). The needed compactness property is then a consequence of the compactness of the embedding $B V(I) \subset L^{2}(I)$, for all bounded intervals $I$. b) The identification of the limit as the entropy solution of (7.1) with initial datum $u_{*}^{0}$. This can be proved using this compactness property and passing to the limit in the variational formulation of (7.1). We refer to [EsVa93] for a detailed description of this limit process in the more delicate case where the initial data converge to a Dirac delta.

This completes the proof of the existence of minimizers in the inviscid case. 
In the viscous one, one cannot use the finite velocity of propagation. However, it is easy to get uniform bounds on the queues of solutions as $|x| \rightarrow \infty$, which allows us to reduce the global compactness problem to a local one. Locally, the same argument as above, based on the one-sided estimate (7.7), which is also true for the viscous equations, applies.

Remark 1. The above proof is in fact quite general and it can be adapted to other optimization problems with different functionals and admissible sets. In particular, using Oleinik's one-sided Lipschitz condition (7.7), one can also consider admissible sets of the form

$$
\mathcal{U}_{a d}=\left\{f \in L^{1}(\mathbb{R}), \operatorname{supp}(f) \subset K,\|f\|_{1} \leq C\right\} .
$$

\subsection{Vanishing Viscosity}

The purpose of this section is to show that the minimizers of the vicous problem $(\nu>0)$ converge to a minimizer of the inviscid problem as the viscosity tends to zero, $\nu \rightarrow 0$.

Theorem 2. Any accumulation point as $\nu \rightarrow 0$ of $u_{\nu}^{0, \text { min }}$, the minimizers of (7.5), with respect to the weak topology in $L^{2}$, is a minimizer of the continuous problem (7.4).

Proof of Theorem 2. We follow a standard $\Gamma$-convergence argument, as in [CaPa08], in the context of the convergence of minimizers for the numerical approximation schemes.

The proof is similar to the one in Theorem 1, although, this time, $\nu \rightarrow 0$.

The key ingredient is the following continuity property. Assume that $u_{\nu}^{0} \in$ $\mathcal{U}_{a d}$ satisfies $u_{\nu}^{0} \rightarrow u^{0}$ weakly in $L^{2}(\mathbb{R})$. Then

$$
J_{\nu}\left(u_{\nu}^{0}\right) \rightarrow J\left(u^{0}\right) .
$$

This is due to the fact that the OSLC condition, together with the uniform $L^{1}$-bound, guarantees uniform local $B V$ bounds on the viscous solutions. For the viscous problem we do not have a finite velocity of propagation property but, as mentioned above, the uniform control of the queues allows us to reduce the compactness problem to a local one and then the local $B V$ bounds suffice.

The limit process, as the viscosity parameter tends to zero, to recover in the limit the weak entropy solution of the inviscid model, can be conducted in a classical way. This is, for instance, done in [EsVa93].

Now, let $\hat{u}^{0} \in \mathcal{U}_{a d}$ be an accumulation point of $u_{\nu}^{0, \text { min }}$ with respect to the weak topology of $L^{2}$. To simplify the notation, we still denote by $u_{\nu}^{0, \text { min }}$ the subsequence for which $u_{\nu}^{0, \min } \rightarrow \hat{u}^{0}$, weakly-* in $L^{\infty}(\mathbb{R})$, as $\nu \rightarrow 0$. Let $v^{0} \in \mathcal{U}_{a d}$ be any other function. We are going to prove that

$$
J\left(\hat{u}^{0}\right) \leq J\left(v^{0}\right) .
$$


To do this we construct a sequence $v_{\nu}^{0} \in \mathcal{U}_{a d}^{\nu}$ such that $v_{\nu}^{0} \rightarrow v^{0}$, in $L^{2}(\mathbb{R})$, as $\nu \rightarrow 0$. In this particular case, taking into account that the set of admissible controls $\mathcal{U}_{a d}^{\nu}$ is independent of $\nu>0$, i.e., $\mathcal{U}_{a d}=\mathcal{U}_{a d}^{\nu}$, it is sufficient to choose, in particular, $v_{\nu}^{0}=v^{0}$.

Taking into account the continuity property (7.8), we have

$$
J\left(v^{0}\right)=\lim _{\nu \rightarrow 0} J_{\nu}\left(v_{\nu}^{0}\right) \geq \lim _{\nu \rightarrow 0} J_{\nu}\left(u_{\nu}^{0, \min }\right)=J\left(\hat{u}^{0}\right),
$$

which proves (7.9).

Remark 2. Theorem 2 concerns global minima. However, both the continuous and discrete functionals may possibly have local minima as well. Extending this kind of $\Gamma$-convergence result for local minima requires important further developments.

\subsection{Sensitivity Analysis: The Inviscid Case}

In this section we collect the results in [CaPa08] for the sensitivity of the functional $J$ in the presence of shocks, which follows previous works, e.g., [BrMa95a], [BaPi02], [BoJa98], [BoJa99], [U103], and [GoRa99].

We focus on the particular case of solutions having a single shock, but the analysis can be extended to consider more general one-dimensional systems of conservation laws with a finite number of noninteracting shocks (see $[$ BrMa95a]).

\subsubsection{Linearization of the Inviscid Burgers Equation}

Following [CaPa08], we introduce the following hypothesis.

(H) Assume that $u(x, t)$ is a weak entropy solution of (7.1) with a discontinuity along a regular curve $\Sigma=\{(t, \varphi(t)), t \in[0, T]\}$, which is Lipschitz continuous outside $\Sigma$. In particular, it satisfies the Rankine-Hugoniot condition on $\Sigma$,

$$
\varphi^{\prime}(t)[u]_{\varphi(t)}=\left[u^{2} / 2\right]_{\varphi(t)},
$$

or, simply,

$$
\varphi^{\prime}(t)=\left(u\left(\varphi(t)^{+}, t\right)+u\left(\varphi(t)^{-}, t\right)\right) / 2 .
$$

Here we have used the notation $[v]_{x_{0}}=v\left(x_{0}^{+}\right)-v\left(x_{0}^{-}\right)$for the jump at $x_{0}$ of any piecewise continuous function $v$ with a discontinuity at $x=x_{0}, v\left(x_{0}^{ \pm}\right)$ standing for the values of $v$ to both sides of $x_{0}$.

Note that $\Sigma$ divides $\mathbb{R} \times(0, T)$ into two parts: $Q^{-}$and $Q^{+}$, the subdomains of $\mathbb{R} \times(0, T)$ to the left and to the right of $\Sigma$, respectively (see Figure 7.1).

As explained in [CaPa08], in the presence of shocks, for correctly dealing with optimal control and design problems, the state of the system (7.1) has 


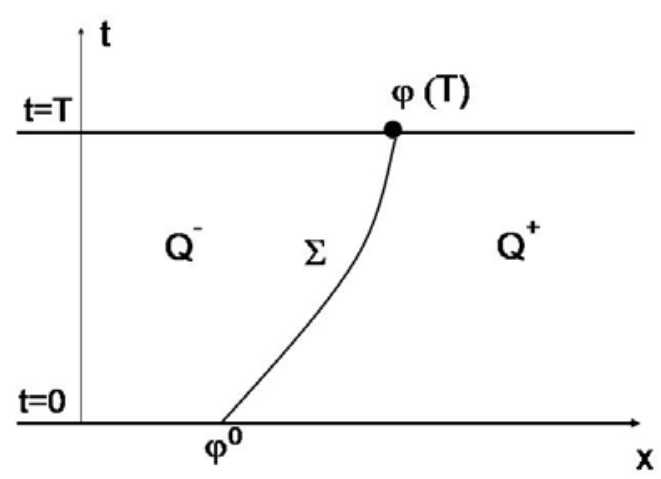

Fig. 7.1. The subdomains $Q^{-}$and $Q^{+}$.

to be viewed as being a pair $(u, \varphi)$ combining the solution of (7.1) and the shock location $\varphi$.

Then the pair $(u, \varphi)$ satisfies the system

$$
\begin{cases}\partial_{t} u+\partial_{x}\left(\frac{u^{2}}{2}\right)=0, & \text { in } Q^{-} \cup Q^{+}, \\ \varphi^{\prime}(t)[u]_{\varphi(t)}=\left[u^{2} / 2\right]_{\varphi(t)}, & t \in(0, T), \\ \varphi(0)=\varphi^{0}, & \text { in }\left\{x<\varphi^{0}\right\} \cup\left\{x>\varphi^{0}\right\} . \\ u(x, 0)=u^{0}(x), & \end{cases}
$$

We now analyze the sensitivity of $(u, \varphi)$ with respect to perturbations of the initial datum, in particular, with respect to variations $\delta u^{0}$ of the initial profile $u^{0}$ and $\delta \varphi^{0}$ of the shock location $\varphi^{0}$. To be precise, we adopt the functional framework based on the generalized tangent vectors introduced in $[\mathrm{BrMa} 95 \mathrm{a}]$.

Definition 1 ([BrMa95a]). Let $v: \mathbb{R} \rightarrow \mathbb{R}$ be a piecewise Lipschitz continuous function with a single discontinuity at $y \in \mathbb{R}$. We define $\Sigma_{v}$ as the family of all continuous paths $\gamma:\left[0, \varepsilon_{0}\right] \rightarrow L^{1}(\mathbb{R})$ with

1. $\gamma(0)=v$ and $\varepsilon_{0}>0$ possibly depending on $\gamma$.

2. For any $\varepsilon \in\left[0, \varepsilon_{0}\right]$ the functions $u^{\varepsilon}=\gamma(\varepsilon)$ are piecewise Lipschitz with a single discontinuity at $x=y^{\varepsilon}$ depending continuously on $\varepsilon$ and there exists a constant $L$ independent of $\varepsilon \in\left[0, \varepsilon_{0}\right]$ such that

$$
\left|v^{\varepsilon}(x)-v^{\varepsilon}\left(x^{\prime}\right)\right| \leq L\left|x-x^{\prime}\right|
$$

whenever $y^{\varepsilon} \notin\left[x, x^{\prime}\right]$.

Furthermore, we define the set $T_{v}$ of generalized tangent vectors of $v$ as the space of $(\delta v, \delta y) \in L^{1} \times \mathbb{R}$ for which the path $\gamma_{(\delta v, \delta y)}$ given by

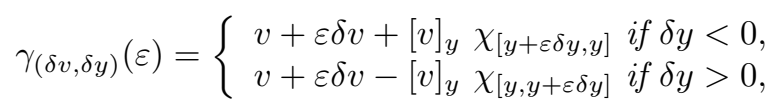


satisfies $\gamma_{(\delta v, \delta y)} \in \Sigma_{v}$.

Finally, we define the equivalence relation $\sim$ defined on $\Sigma_{v}$ by

$$
\gamma \sim \gamma^{\prime} \text { if and only if } \lim _{\varepsilon \rightarrow 0} \frac{\left\|\gamma(\varepsilon)-\gamma^{\prime}(\varepsilon)\right\|_{L^{1}}}{\varepsilon}=0,
$$

and we say that a path $\gamma \in \Sigma_{v}$ generates the generalized tangent vector $(\delta v, \delta y) \in T_{v}$ if $\gamma$ is equivalent to $\gamma_{(\delta v, \delta y)}$ as in (7.13).

Remark 3. The path $\gamma_{(\delta v, \delta y)} \in \Sigma_{v}$ in (7.13) represents, at first order, the variation of a function $v$ by adding a perturbation function $\varepsilon \delta v$ and by shifting the discontinuity by $\varepsilon \delta y$.

Note that, for a given $v$ (piecewise Lipschitz continuous function with a single discontinuity at $y \in \mathbb{R}$ ) the associated generalized tangent vectors $(\delta v, \delta y) \in T_{v}$ are those pairs for which $\delta v$ is Lipschitz continuous with a single discontinuity at $x=y$.

Let $u^{0}$ be the initial datum in (7.12) that we assume to be Lipschitz continuous to both sides of a single discontinuity located at $x=\varphi^{0}$, and consider a generalized tangent vector $\left(\delta u^{0}, \delta \varphi^{0}\right) \in L^{1}(\mathbb{R}) \times \mathbb{R}$. Let $u^{0, \varepsilon} \in \Sigma_{u^{0}}$ be a path which generates $\left(\delta u^{0}, \delta \varphi^{0}\right)$. For $\varepsilon$ sufficiently small the solution $u^{\varepsilon}(\cdot, t)$ of $(7.12)$ is Lipschitz continuous with a single discontinuity at $x=$ $\varphi^{\varepsilon}(t)$, for all $t \in[0, T]$. Thus, $u^{\varepsilon}(\cdot, t)$ generates a generalized tangent vector $(\delta u(\cdot, t), \delta \varphi(t)) \in L^{1}(\mathbb{R}) \times \mathbb{R}$. Moreover, in [BrMa95b] it is proved that it satisfies the following linearized system:

$$
\left\{\begin{array}{l}
\partial_{t} \delta u+\partial_{x}(u \delta u)=0, \quad \text { in } Q^{-} \cup Q^{+} \\
\delta \varphi^{\prime}(t)[u]_{\varphi(t)}+\delta \varphi(t)\left(\varphi^{\prime}(t)\left[u_{x}\right]_{\varphi(t)}-\left[u_{x} u\right]_{\varphi(t)}\right) \\
\quad+\varphi^{\prime}(t)[\delta u]_{\varphi(t)}-[u \delta u]_{\varphi(t)}=0, \quad \text { in }(0, T), \\
\delta u(x, 0)=\delta u^{0}, \quad \text { in }\left\{x<\varphi^{0}\right\} \cup\left\{x>\varphi^{0}\right\}, \\
\delta \varphi(0)=\delta \varphi^{0},
\end{array}\right.
$$

with the initial data $\left(\delta u^{0}, \delta \varphi^{0}\right)$.

Remark 4. In this way, we can obtain formally the expansion

$$
\left(u_{\varepsilon}, \varphi_{\varepsilon}\right)=(u, \varphi)+\varepsilon(\delta u, \delta \varphi)+\mathcal{O}\left(\varepsilon^{2}\right) .
$$

Remark 5. The linearized system (7.14) has a unique solution which can be computed in two steps. The method of characteristics determines $\delta u$ in $Q^{-} \cup Q^{+}$, i.e., outside $\Sigma$, from the initial data $\delta u^{0}$ (note that system (7.14) has the same characteristics as (7.12)). This yields the value of $u$ and $u_{x}$ at both sides of the shock $\Sigma$ and allows the determination of the coefficients of the ordinary different equation (ODE) that $\delta \varphi$ satisfies. This ODE yields $\delta \varphi$.

Remark 6 . We have assumed that the discontinuity of the solution of the Burgers equation $u$ is present in the whole time interval $t \in[0, T]$. But the discontinuities may appear at time $\tau \in(0, T)$ for some regular initial data. We refer to $[\mathrm{CaPa} 08]$ for the linearization in this case. 


\subsubsection{Sensitivity in the Presence of Shocks}

In this section we study the sensitivity of the functional $J$ with respect to variations associated with the generalized tangent vectors defined in the previous section. We first define an appropriate generalization of the Gateaux derivative.

Definition 2 ([BrMa95a]). Let $J: L^{1}(\mathbb{R}) \rightarrow \mathbb{R}$ be a functional and $u^{0} \in$ $L^{1}(\mathbb{R})$ be Lipschitz continuous with a discontinuity at $x=\varphi^{0}$, an initial datum for which the solution of (7.1) satisfies hypothesis (H). We say that $J$ is Gateaux differentiable at $u^{0}$ in a generalized sense if for any generalized tangent vector $\left(\delta u^{0}, \delta \varphi^{0}\right)$ and any family $u^{0, \epsilon} \in \Sigma_{u^{0}}$ associated to $\left(\delta u^{0}, \delta \varphi^{0}\right)$ the following limit exists:

$$
\delta J=\lim _{\epsilon \rightarrow 0} \frac{J\left(u^{0, \epsilon}\right)-J\left(u^{0}\right)}{\epsilon},
$$

and it depends only on $\left(u^{0}, \varphi^{0}\right)$ and $\left(\delta u^{0}, \delta \varphi^{0}\right)$, i.e., it does not depend on the particular family $u^{0, \epsilon}$ which generates $\left(\delta u^{0}, \delta \varphi^{0}\right)$.

The limit $\delta J$ is the generalized Gateux derivative of $J$ in the direction $\left(\delta u^{0}, \delta \varphi^{0}\right)$.

The following result provides an easy characterization of the generalized Gateaux derivative of $J$ in terms of the solution of the associated adjoint system.

Proposition 1. The Gateaux derivative of $J$ can be written as

$$
\delta J=\int_{\left\{x<\varphi^{0}\right\} \cup\left\{x>\varphi^{0}\right\}} p(x, 0) \delta u^{0}(x) d x+q(0)\left[u^{0}\right]_{\varphi^{0}} \delta \varphi^{0},
$$

where the adjoint state pair $(p, q)$ satisfies the system

$$
\left\{\begin{array}{l}
-\partial_{t} p-u \partial_{x} p=0, \quad \text { in } Q^{-} \cup Q^{+}, \\
{[p]_{\Sigma}=0,} \\
q(t)=p(\varphi(t), t), \text { in } t \in(0, T) \\
q^{\prime}(t)=0, \text { in } t \in(0, T) \\
p(x, T)=u(x, T)-u^{d}, \quad \text { in }\{x<\varphi(T)\} \cup\{x>\varphi(T)\} \\
q(T)=\frac{\frac{1}{2}\left[\left(u(x, T)-u^{d}\right)^{2}\right]_{\varphi(T)}}{[u]_{\varphi(T)}} .
\end{array}\right.
$$

Remark 7. System (7.16) has a unique solution. In fact, to solve the backwards system (7.16) we first define the solution $q$ on the shock $\Sigma$ from the condition $q^{\prime}=0$, with the final value $q(T)$ given in (7.16). This determines the value of $p$ along the shock. We then propagate this information, together with the datum of $p$ at time $t=T$ to both sides of $\varphi(T)$, by characteristics. As both systems (7.1) and (7.16) have the same characteristics, any point $(x, t) \in$ $\mathbb{R} \times(0, T)$ is reached backwards in time by a unique characteristic line coming 
either from the shock $\Sigma$ or the final data at $(x, T)$ (see Figure 7.2). The solution obtained this way coincides with the reversible solutions introduced in [BoJa98] and [BoJa99].
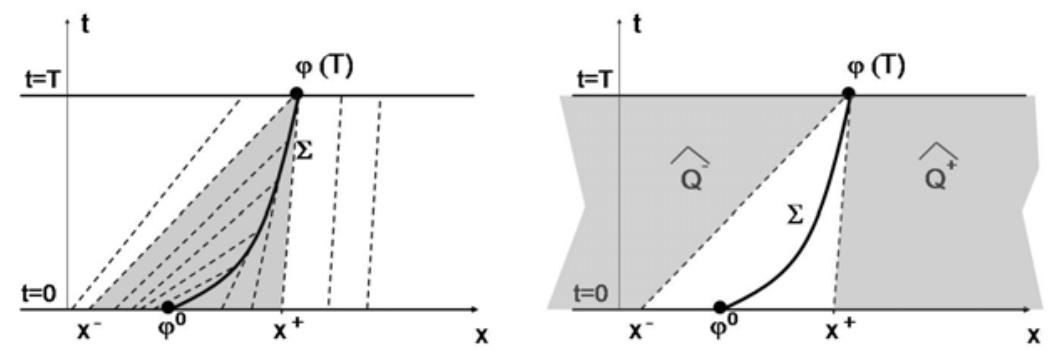

Fig. 7.2. Characteristic lines entering on a shock (left) and subdomains $\hat{Q}^{-}$and $\hat{Q}^{+}$(right).

Remark 8. Solutions of (7.16) can also be obtained as the limit of solutions of the transport equation with artificial viscosity depending on a small parameter $\varepsilon \rightarrow 0$,

$$
\left\{\begin{array}{l}
-\partial_{t} p-u \partial_{x} p=\varepsilon \partial_{x x} p, \quad \text { in } x \in \mathbb{R}, t \in(0, T), \\
p(x, T)=p_{n}^{T}(x), \quad \text { in } x \in \mathbb{R}
\end{array}\right.
$$

and a suitable choice of the initial data $p_{n}^{T}(x)$, depending on $n \rightarrow \infty$. To be more precise, let $p_{n}^{T}(x)$ be any sequence of Lipschitz continuous functions, uniformly bounded in $B V_{\text {loc }}(\mathbb{R})$, such that

$$
p_{n}^{T}(x, T) \rightarrow p^{T}(x)=u(x, T)-u^{d}(x), \quad \text { in } L_{l o c}^{1}(\mathbb{R}),
$$

and

$$
p_{n}^{T}(\varphi(T), T)=\frac{\frac{1}{2}\left[\left(u(x, T)-u^{d}\right)^{2}\right]_{\varphi(T)}}{[u]_{\varphi(T)}} .
$$

We first take the limit of the solutions $p_{\varepsilon, n}$ of (7.17) as $\varepsilon \rightarrow 0$, to obtain the solution $p_{n}$ of

$$
\left\{\begin{array}{l}
-\partial_{t} p-u \partial_{x} p=0, \quad \text { in } x \in \mathbb{R}, t \in(0, T), \\
p(x, T)=p_{n}^{T}(x), \quad \text { in } x \in \mathbb{R},
\end{array}\right.
$$

which is called the reversible solution (see [BoJa98]). These solutions can be characterized by the fact that they take the value $p_{n}(\varphi(T), T)$ in the whole region occupied by the characteristics that meet the shock (see [BoJa98], Theorem 4.1.12). Thus, in particular, they satisfy the 2nd, 3rd, 4th, and 6th equations in (7.16). Moreover, $p_{n} \rightarrow p$ as $n \rightarrow \infty$, and $p$ takes a constant value 

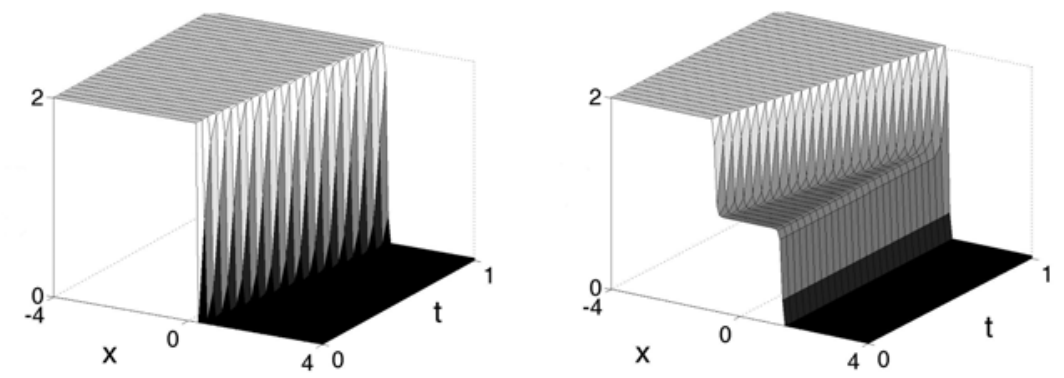

Fig. 7.3. Solution $u(x, t)$ of the Burgers equation with an initial datum having a discontinuity (left) and adjoint solution which takes a constant value in the region occupied by the characteristics that meet the shock (right).

in the region occupied by the characteristics that meet the shock. Note that, by construction, this constant is the same value for all $p_{n}$ in this region. Thus, this limit solution $p$ coincides with the solution of (7.16) constructed above.

Formula (7.15) provides an obvious way to compute a first descent direction of $J$ at $u^{0}$. We just take

$$
\left(\delta u^{0}, \delta \varphi^{0}\right)=\left(-p(x, 0),-q(0)[u]_{\varphi^{0}}\right) .
$$

Here, the value of $\delta \varphi^{0}$ must be interpreted as the optimal infinitesimal displacement of the discontinuity of $u^{0}$.

But this $\left(\delta u^{0}, \delta \varphi^{0}\right)$ is not a generalized tangent vector in $T_{u^{0}}$ since $p(x, 0)$ is not continuous away from $x \neq \varphi^{0}$. A typical example is shown in Figure 7.3. In [CaPa08] we have solved this drawback by introducing the alternating descent algorithm, which only uses generalized tangent vectors, distinguishing those that move the shock and those that do not.

\subsection{The Method of Alternating Descent Directions}

In this section we briefly present the alternating descent algorithm introduced in $[\mathrm{CaPa} 08]$ in the inviscid case.

Motivated by the above discussion, we introduce a decomposition of the generalized tangent vectors. This requires us first to introduce some notation. Let

$$
x^{-}=\varphi(T)-u^{-}(\varphi(T)) T, \quad x^{+}=\varphi(T)-u^{+}(\varphi(T)) T,
$$

and consider the following subsets (see Figure 7.2):

$$
\hat{Q}^{-}=\left\{(x, t) \in \mathbb{R} \times(0, T) \text { such that } x<\varphi(T)-u^{-}(\varphi(T)) t\right\},
$$




$$
\hat{Q}^{+}=\left\{(x, t) \in \mathbb{R} \times(0, T) \text { such that } x>\varphi(T)-u^{+}(\varphi(T)) t\right\} .
$$

The classification of the generalized tangent vectors in two classes is motivated by the following result (see [CaPa08]).

Proposition 2. Consider the paths in $\Sigma_{u^{0}}$ for which the associated generalized tangent vectors $\left(\delta u^{0}, \delta \varphi^{0}\right) \in T_{u^{0}}$ satisfy

$$
\delta \varphi^{0}=-\frac{\int_{x^{-}}^{\varphi^{0}} \delta u^{0}+\int_{\varphi^{0}}^{x^{+}} \delta u^{0}}{\left[u^{0}\right]_{\varphi^{0}}} .
$$

Then, the solution $(\delta u, \delta \varphi)$ of system (7.14) satisfies $\delta \varphi(T)=0$ and the generalized Gateaux derivative of $J$ in the direction $\left(\delta u^{0}, \delta \varphi^{0}\right)$ can be written as

$$
\delta J=\int_{\left\{x<x^{-}\right\} \cup\left\{x>x^{+}\right\}} p(x, 0) \delta u^{0}(x) d x,
$$

where $p$ satisfies the system

$$
\left\{\begin{array}{l}
-\partial_{t} p-u \partial_{x} p=0, \quad \text { in } \hat{Q}^{-} \cup \hat{Q}^{+} \\
p(x, T)=u(x, T)-u^{d}, \quad \text { in }\{x<\varphi(T)\} \cup\{x>\varphi(T)\} .
\end{array}\right.
$$

Analogously, when considering paths in $\Sigma_{u^{0}}$ for which the associated generalized tangent vectors $\left(\delta u^{0}, \delta \varphi^{0}\right) \in T_{u^{0}}$ satisfy $\delta u^{0}=0$, then $\delta u(x, T)=0$ and the generalized Gateaux derivative of $J$ in the direction $\left(\delta u^{0}, \delta \varphi^{0}\right)$ can be written as

$$
\delta J=-\left[\frac{\left(u(x, T)-u^{d}(x)\right)^{2}}{2}\right]_{\varphi(T)} \frac{\left[u^{0}\right]_{\varphi^{0}}}{[u(\cdot, T)]_{\varphi(T)}} \delta \varphi^{0} .
$$

Remark 9. Formula (7.21) provides a simplified expression for the generalized Gateaux derivative of $J$ when considering directions $\left(\delta u^{0}, \delta \varphi^{0}\right)$ that do not move the shock position at $t=T$. These directions are characterized by formula (7.20) which determines the infinitesimal displacement of the shock position $\delta \varphi^{0}$ in terms of the variation of $u^{0}$ to both sides of $x=\varphi^{0}$. Note, in particular, that to any value $\delta u^{0}$ to both sides of the jump $\varphi^{0}$ there corresponds a unique infinitesimal translation $\delta \varphi^{0}$ of the initial shock position that does not move it at $t=T$.

Note also that the value of $p$ outside the region $\hat{Q}^{-} \cup \hat{Q}^{+}$is not needed to evaluate the generalized Gateaux derivative in (7.21). Solving system (7.22) is particularly easy since the potential $u$ is smooth in the region where the system is formulated.

Analogously, formula (7.23) provides a simplified expression of the generalized Gateaux derivative of $J$ when considering directions $\left(\delta u^{0}, \delta \varphi^{0}\right)$ that uniquely move the shock position at $t=T$ and which correspond to purely translating the shock. 
The method of alternating descent directions can then be implemented as follows, applying in each step of the descent, the following two substeps:

1. Use generalized tangent vectors that move the shock to search its optimal placement.

2. Use generalized tangent vectors to modify the value of the solution at time $t=T$ to both sides of the discontinuity, leaving the shock location unchanged.

One of the main advantages of this method is that the complexity of the solutions is preserved without introducing artificial shocks that are unnecessary to approximate the target $u^{d}$.

The efficiency of this method compared to the classical one based on purely discrete approaches or continuous ones that do not make an optimal use of the shock analysis has been illustrated in [CaPa08] through several numerical experiments.

Note also that this method is, in some sense, close to the methods employed in shape design in elasticity in which topological derivatives (that in the present setting would correspond to controlling the location of the shock) are combined with classical shape deformations (that would correspond to simply shaping the solution away from the shock in the present setting) ([GaGu01]).

\subsection{Alternating Descent Directions in the Viscous Case}

The linearized Burgers equation reads as follows:

$$
\left\{\begin{array}{l}
\partial_{t} \delta u-\nu \delta u_{x x}+\partial_{x}(u \delta u)=0, \quad \text { in } \mathbb{R} \times(0, \infty), \\
\delta u(x, 0)=\delta u^{0}, \quad \text { in } \mathbb{R} .
\end{array}\right.
$$

In this case the derivation of this linearized equation is straightforward because of the smoothness of solutions.

Moreover, the Gateaux derivative of the functional $J$ is as follows:

$$
\delta J=<\delta J\left(u^{0}\right), \delta u^{0}>=\int_{\mathbb{R}} p(x, 0) \delta u^{0}(x) d x,
$$

where the adjoint state $p=p_{\nu}$ solves the adjoint system

$$
\left\{\begin{array}{l}
-\partial_{t} p-\nu p_{x x}-u \partial_{x} p=0, \quad \text { in } \mathbb{R}, 0<t<T, \\
p(x, T)=u(x, T)-u^{d}, \quad \text { in } \mathbb{R} .
\end{array}\right.
$$

In this case, unlike the inviscid one, the adjoint state has only one component. Indeed, since the state does not present shocks, there is no adjoint shock variable. Similarly, the derivative of $J$ in (7.15) exhibits only one term. According to this, the straightforward application of a gradient method for the optimization of $J$ would lead, in each step of the iteration, to the use of variations pointing in the direction 


$$
\delta u^{0}=-p(x, 0),
$$

$p$ being the solution of this viscous adjoint system. But, proceeding in this way, we would not exploit the possibilities that the alternate descent method provides.

To do this we must also consider the effect of possible infinitesimal translations of the initial data. Indeed, the previous calculus is valid when the variations of the initial data are of the form

$$
u_{\varepsilon}^{0}(x)=u^{0}(x)+\varepsilon \delta u^{0}(x) .
$$

But, in order to consider the possible effect of the infinitesimal translations, we have to use rather variations of the form

$$
u_{\varepsilon}^{0}(x)=u^{0}\left(x+\varepsilon \delta \varphi^{0}\right)+\varepsilon \delta u^{0},
$$

where, now, $\varphi^{0}$ stands for a reference point on the profile of $u^{0}$, not necessarily a point of discontinuity. When $u^{0}$ has a point of discontinuity, $\varphi^{0}$ could be its location and $\delta \varphi^{0}$ an infinitesimal variation of it. But $\varphi^{0}$ could also stand for another singular point on the profile of $u^{0}$, e.g., an extremal point, or a point where the gradient of $u^{0}$ is large, i.e., a smeared discontinuity.

Note that, by a Taylor expansion, when considering variations of this form, to first order, this corresponds to

$$
u_{\varepsilon}^{0}(x) \sim u^{0}(x)+\varepsilon \delta u^{0}(x)+\varepsilon \delta \varphi^{0} u_{x}^{0}(x) .
$$

This indicates that the result of these combined variations $\left(\delta u^{0}, \delta \varphi^{0}\right)$ is equivalent to a classical variation in the direction of $\delta u^{0}+\delta \varphi^{0} u_{x}^{0}$. When $u^{0}$ is smooth enough, for instance, $u^{0} \in H^{1}$, then, this yields a standard variation in an $L^{2}$ direction. But when $u^{0}$ lacks regularity, for instance, when $u^{0}$ has a point of discontinuity, this yields variations that are singular and contain Dirac deltas. Similarly, when $u^{0}$ is smooth but has a large gradient, we see that the effect of a small $\delta \varphi^{0}$ is amplified by the size of the gradient.

The corresponding linearization of the Burgers equation is as follows:

$$
\left\{\begin{array}{l}
\partial_{t} \delta u-\nu \delta u_{x x}+\partial_{x}(u \delta u)=0, \quad \text { in } \mathbb{R} \times(0, \infty), \\
\delta u(x, 0)=\delta u^{0}(x)+\delta \varphi^{0} u_{x}^{0}(x), \quad \text { in } \mathbb{R} .
\end{array}\right.
$$

Again, the derivation of this linearized equation is straightforward because of the smoothness of solutions.

In view of (7.30) the linearization of the functional is as follows:

$$
\delta J=\int_{\mathbb{R}} p(x, 0) \delta u^{0}(x) d x+\delta \varphi^{0} \int_{\mathbb{R}} p(x, 0) u_{x}^{0}(x) d x,
$$

where the adjoint state $p=p_{\nu}$ is as above.

When $u^{0}$ is piecewise smooth but it has a discontinuity at $x=\varphi^{0}$, this variation can be written as follows: 
$\delta J=\int_{\mathbb{R}} p(x, 0) \delta u^{0}(x) d x+\delta \varphi^{0} \int_{\mathbb{R}-\left\{\varphi^{0}\right\}} p(x, 0) u_{x}^{0}(x) d x+\delta \varphi^{0}\left[u^{0}\right]_{x=\varphi^{0}} p\left(\varphi^{0}, 0\right)$,

where $\left[u^{0}\right]_{x=\varphi^{0}}$ stands for the jump of $u^{0}$ at $x=\varphi^{0}$.

When comparing this expression with (7.15), we see that there is an extra term, namely,

$$
\delta \varphi^{0} \int_{\mathbb{R}-\left\{\varphi^{0}\right\}} p(x, 0) u_{x}^{0}(x) d x,
$$

which corresponds to the fact that the variations considered in the inviscid case by means of the generalized tangents and (7.30) only coincide with those considered here when $u_{0}$ is piecewise constant with a shock at $\varphi^{0}$. When the initial datum has a discontinuity at $x=\varphi^{0}$, a slight change in the way the variations (7.28) are defined, considering the vectors in (7.13), leads to an expression which is closer to (7.15). This is done translating the point of discontinuity by adding as in (7.13) a characteristic function of the amplitude of the jump of $u^{0}$ so that the jump point is shifted infinitesimally to the left or to the right, but without adding any extra variation on the initial profile $u^{0}$ due to this shift.

But, let us continue our analysis by keeping the class of variations (7.28), supposing that $u^{0}$ is continuous. We can rewrite the first variation of $J$ as follows:

$$
\delta J=\int_{\mathbb{R}} p(x, 0)\left[\delta u^{0}(x)+\delta \varphi^{0} u_{x}^{0}(x)\right] d x .
$$

In the inviscid case, to develop the method of alternating descent, we distinguished the variations of the initial datum moving the shock and those that did not move it by modifying the profile away from it. This discussion does not make sense as such in the present setting since the solutions of the viscous state equation are now smooth. However, from a computational viewpoint, it is interesting to develop the analogue of the alternating descent method.

For this to be done, one needs to distinguish two classes of possible variations. But this time one has to do it without having, as in the inviscid case, the shock location and its region of influence at $t=0$ which, in that case, we identified with the interval $\left[x^{-}, x^{+}\right]$as in (7.19).

Let us however assume that the viscosity parameter $\nu$ is small enough, so that viscous solutions are close to the inviscid ones, and develop a strategy inspired in the way that the alternating descent direction was built in the inviscid case. For it to be meaningful, we need to identify a class of initial data for which the alternating descent method might be more efficient than the classical one, which consists in simply applying a descent algorithm based on the adjoint calculus above. We will identify this class as the one in which the initial data $u^{0}$ leads to a discontinuous solution in the inviscid case.

Assume, to begin with, that $u^{0}$ has a discontinuity at $\varphi^{0}$ and that it is smooth to both sides of it. The viscosity parameter $\nu$ being positive, even if 
it is small, the solution is smooth and, therefore, it may not develop shocks. However, taking into account that solutions are close to the inviscid ones, when the latter exhibit shocks, the viscous ones will develop regularized quasishocks. Therefore, one could try to mimic the same procedure for the viscous case. The first thing to be done is to identify the region of influence $\left[x^{-}, x^{+}\right]$ of the inner boundary of the inviscid adjoint system. But, of course, this should be done without solving the inviscid adjoint system which, on the other hand, would require solving the inviscid state equation. We therefore need an alternate definition of the interval $\left[x^{-}, x^{+}\right]$to that in (7.19) which might be easy to compute. To do that it is necessary to compute the curve where the shock is located in the inviscid case. This can be done by solving the ODE given by the Rankine-Hugoniot condition:

$$
\varphi^{\prime}(t)=\left[u^{+}(\varphi(t), t)+u^{-}(\varphi(t), t)\right] / 2, \quad t \in(0, T) .
$$

Here $u^{+}$and $u^{-}$stand for the value of the solution $u$ of the inviscid problem at both sides of the shock. They can be computed by the method of characteristics so that

$$
u^{ \pm}(\varphi(t), t)=u^{0}\left(s^{ \pm}(t)\right), \quad t \in(0, T),
$$

where $s^{ \pm}(t)$ is the spatial trajectory of the characteristic which arrives to $(\varphi(t), t)$ starting from $t=0$, and we solve

$$
s^{ \pm}(t)+t u^{0, \pm}\left(s^{ \pm}(t)\right)=\varphi(t), \quad t \in(0, T) .
$$

Substituting (7.35) and (7.36) into (7.34), the ODE for the shock then reads

$$
\varphi^{\prime}(t)=\left[u^{0}\left(s^{+}(t)\right)+u^{0}\left(s^{-}(t)\right)\right] / 2, \quad t \in(0, T),
$$

and

$$
x^{ \pm}=s^{ \pm}(T) .
$$

Once this is done, we need to identify the variations $\left(\delta u^{0}, \delta \varphi^{0}\right)$ such that

$$
\int_{x^{-}}^{x^{+}} p(x, 0)\left[\delta u^{0}(x)+\delta \varphi^{0} u_{x}^{0}(x)\right] d x=0 .
$$

If $p(x, 0)$ were constant within the interval $\left[x^{-}, x^{+}\right]$as in the inviscid case, this would amount to considering variations such that

$$
\delta \varphi^{0}=-\frac{\int_{x^{-}}^{x^{+}} \delta u^{0}(x) d x}{u^{0}\left(x^{+}\right)-u^{0}\left(x^{-}\right)} .
$$

There is no unique way of doing this. One possibility would be to consider variations $\delta u^{0}$ in $\left[x^{-}, x^{+}\right]$such that $\int_{x^{-}}^{x^{+}} \delta u^{0}(x) d x=0$ and $\delta \varphi^{0}=0$.

The variation of the functional $J$ would then be 


$$
\delta J=\int_{\left\{x<x^{-}\right\} \cap\left\{x>x^{+}\right\}} p(x, 0) \delta u^{0}(x) d x,
$$

and the optimal descent direction

$$
\delta u^{0}(x)=-p(x, 0), \quad \text { in }\left\{x<x^{-}\right\} \cap\left\{x>x^{+}\right\} .
$$

This discussion leads to considering "descent directions" of the form (7.42), where $p$ is the solution of the adjoint viscous system and the extremes of the interval $x^{ \pm}$are computed according to (7.36)-(7.38). Furthermore, $\delta u^{0}$ has to be extended to $\left[x^{-}, x^{+}\right]$so that $\int_{x^{-}}^{x^{+}} \delta u^{0}(x) d x=0$ and $\delta \varphi^{0}=0$. Note also that, as observed in [CaPa08], it is convenient to choose $\delta u^{0}$ which is continuous away from $\varphi^{0}$ to guarantee that the deformations under consideration do not increase the number of possible discontinuities of $u^{0}$. Obviously, this is possible within the class of variations we have identified.

This class of deformations has been identified under the assumption that $p(x, 0)$ is constant within the interval $\left[x^{-}, x^{+}\right]$, a property that is indeed true in the inviscid case but not in the viscous one. The rigorous analysis of the convergence of the adjoint states as the viscosity parameter $\nu$ tends to zero, and the possible improvement of the class of variations above, is an interesting topic for future research.

The second class of variations is the one that takes advantage of the infinitesimal translations $\delta \varphi^{0}$. We can then set $\delta u^{0} \equiv 0$ and choose $\delta \varphi^{0}$ such that

$$
\delta \varphi^{0}=-\int_{\mathbb{R}-\left\{\varphi^{0}\right\}} p(x, 0) u_{x}^{0}(x) d x-\left[u^{0}\right]_{x=\varphi^{0}} p\left(\varphi^{0}, 0\right) .
$$

As mentioned above, we could consider slightly different variations of the initial data of the form

$$
\delta \varphi^{0}=-\left[u^{0}\right]_{x=\varphi^{0}} p\left(\varphi^{0}, 0\right)
$$

as in [CaPa08].

On the other hand, in the inviscid case, $p\left(\varphi^{0}, 0\right)$ coincides with the value of $p$ at time $t=T$ at the shock location and, therefore, this descent direction can be computed without performing any numerical approximation of $p$. This is no longer the case in the present viscous setting in which $p\left(\varphi^{0}, 0\right)$ is a priori unknown. To simplify the choice, we can use the proximity of the inviscid adjoint state and the viscous one. When doing this and using the above (slightly different) variations of the initial data, the choice for $\delta \varphi^{0}$, inspired by $(7.23)$, would be

$$
\delta \varphi^{0}=\left[\frac{\left(u(x, T)-u^{d}(x)\right)^{2}}{2}\right]_{\varphi(T)} \frac{\left[u^{0}\right]_{\varphi^{0}}}{[u(\cdot, T)]_{\varphi(T)}},
$$

where $\varphi(T)$ is the location of the shock in the inviscid case which, in view of (7.36)-(7.38), is given by

$$
\varphi(T)=x^{-}+T u^{0}\left(x^{-}\right)=x^{+}+T u^{0}\left(x^{+}\right) .
$$


Similarly, in the inviscid case, the computation of the jump of $u(\cdot, T)$ and $\left(u(x, T)-u^{d}(x)\right)^{2}$ at $x=\varphi(T)$ can be greatly simplified since the values of $u$ at $t=T$ at both sides of the discontinuity $x=\varphi(T)$ can be computed by the method of characteristics and coincide with $u^{0}\left(x^{ \pm}\right)$.

In this way, we have identified two classes of variations and its approximate values inspired in the structure of the state and the adjoint state in the inviscid case, allowing us to implement the method of alternating descent in the inviscid case when $u^{0}$ is discontinuous.

This analysis can be extended to the case where $u^{0}$ is smooth but the corresponding solution of the inviscid problem develops shock discontinuities in some time $0 \leq \tau<T$. This can be fully characterized in terms of $u^{0}$, as is well known. Then, the analysis of the previous case can be applied with the possible variant discussed in $[\mathrm{CaPa} 08]$ when the shock does not start at $t=0$ but rather appears in a time $0<\tau<T$.

In this way one can handle, for instance, the prototypical solutions of the viscous Burgers equation that, as $\nu \rightarrow 0$, converge to shock solutions ([Wh74]). These are the smooth traveling wave solutions of the viscous Burgers equation (7.2) taking values $u_{ \pm}$at $\pm \infty$, of the form,

$$
u_{\nu}(x, t)=u_{+}+\frac{u_{-}-u_{+}}{1+\exp \left[\left(u_{-}-u_{+}\right)(x-\bar{u} t) / 2 \nu\right]},
$$

where

$$
\bar{u}=\left(u_{-}+u_{+}\right) / 2 .
$$

When $u_{-}>u_{+}$and $\nu \rightarrow 0$, they converge to the shock solution of the inviscid Burgers equation taking values $u_{+}$for $x>\bar{u} t$ and $u_{-}$for $x<\bar{u} t$.

The efficiency of the method developed in this section is illustrated by several numerical experiments in the following section.

\subsection{Numerical Experiments}

In this section we focus on the numerical approximation of the optimization problem described in this chapter. The first natural question is the choice of the numerical method to approximate both the Burgers equation and its adjoint.

Let us introduce a mesh in $\mathbb{R} \times[0, T]$ given by $\left(x_{j}, t^{n}\right)=(j \Delta x, n \Delta t)$ $(j=-\infty, \ldots, \infty ; n=0, \ldots, N+1$ so that $(N+1) \Delta t=T)$, and let $u_{j}^{n}$ be a numerical approximation of $u\left(x_{j}, t^{n}\right)$ obtained as a solution of a suitable discretization of the Burgers equation.

As we are assuming the viscosity parameter $\nu$ to be small, it seems natural to consider a viscous perturbation of the most common numerical schemes for conservation laws. Accordingly, let us introduce a 3-point conservative numerical approximation scheme for the nonlinearity and an explicit scheme for the viscosity: 


$$
\begin{aligned}
u_{j}^{n+1}= & u_{j}^{n}-\frac{\Delta t}{\Delta x}\left(g_{j+1 / 2}^{n}-g_{j-1 / 2}^{n}\right)+\nu \frac{\Delta t}{\Delta x^{2}}\left(u_{j-1}^{n}-2 u_{j}^{n}+u_{j+1}^{n}\right), \\
& j \in \mathbb{Z}, n=0, \ldots, N,
\end{aligned}
$$

where

$$
g_{j+1 / 2}^{n}=g\left(u_{j}^{n}, u_{j+1}^{n}\right),
$$

and $g$ is the numerical flux. These schemes are consistent with the viscous Burgers equation when $g(u, u)=u^{2} / 2$ since, in this case, both the nonlinear part and the viscous perturbation are consistent.

In order to analyze the scheme (7.45), we note that it can be written as a conservative numerical scheme with the modified numerical flux,

$$
g_{v i s}(u, v)=g(u, v)-\frac{\nu}{\Delta x}(v-u) .
$$

In particular, the stability analysis can be obtained from the classical analysis for conservative schemes.

It is interesting to observe that the stability of these numerical schemes is not granted from the stability of the underlying conservative scheme for the inviscid Burgers equation. To clarify this issue, we divide the rest of this section into two more subsections. In the first one we analyze the stability of the numerical schemes written in the form (7.45) and we introduce a convergent numerical scheme. The second subsection is devoted to illustrate the numerical results for the optimization problem.

\subsubsection{Discussion of the Stability of the Viscous Versions of Hyperbolic Conservative Schemes}

We first focus on the von Neumann analysis for the stability of the simpler linear equation,

$$
u_{t}+a u_{x}=\nu u_{x x}, \quad \text { with } a \text { constant. }
$$

We follow the analysis in [GoRa91] for conservative schemes. It is well known that any 3-point conservative numerical scheme can be written in viscosity form as

$$
u_{j}^{n+1}=u_{j}^{n}-\frac{\Delta t}{\Delta x} a \frac{u_{j+1}^{n}-u_{j-1}^{n}}{2}+\tilde{q} \frac{u_{j+1}^{n}-2 u_{j}^{n}+u_{j-1}^{n}}{2},
$$

for some viscosity coefficient $\tilde{q}$. Therefore, the numerical scheme (7.45) can also be written as (7.48) with the new viscosity coefficient,

$$
q=\tilde{q}+2 \nu \frac{\Delta t}{\Delta x^{2}}
$$

Taking into account that $u_{j+1}^{n} \sim u\left(x_{j}+\Delta x, t_{n}\right)$, if we write $x_{j}=x$ and consider the Fourier transform in $x$, we obtain 


$$
\widehat{u}^{n+1}(\eta)=h(\eta) \widehat{u}^{n}(\eta) .
$$

The value $h(\eta)$ represents the amplification factor that must be smaller than one in modulus to guarantee stability. In this case,

$$
h(\eta)=1-q(1-\cos \eta \Delta x)-i \frac{\Delta t}{\Delta x} a \sin \eta \Delta x .
$$

If we write

$$
y=\sin (\eta \Delta x / 2)^{2}
$$

then

$$
|h(\eta)|^{2}=(1-2 q y)^{2}+4\left(\frac{\Delta t}{\Delta x} a\right)^{2} y(1-y) .
$$

It is not difficult to show that a necessary and sufficient condition for the $L^{2}$-stability, i.e., $|h(\eta)| \leq 1$, is to have

$$
\frac{\Delta t^{2}}{\Delta x^{2}} \leq q=\tilde{q}+2 \nu \frac{\Delta t}{\Delta x^{2}} \leq 1
$$

From this condition we easily deduce that not all convergent numerical methods for solving the inviscid Burgers equation are stable when adding the dissipative term $\nu \frac{\Delta t}{\Delta x^{2}}\left(u_{j-1}^{n}-2 u_{j}^{n}+u_{j+1}^{n}\right)$, even for arbitrarily small $\Delta t$. For example, in the Lax-Friedrichs scheme the numerical flux is given by

$$
g^{l f}(u, v)=a \frac{u+v}{2}-\frac{v-u}{2 \Delta t / \Delta x},
$$

and $\tilde{q}=1$. Therefore, it becomes unstable as soon as $\nu>0$, whatever the choice of $\Delta t$ is.

In the following experiments we have chosen the numerical flux associated to the Engquist-Osher scheme. For the linear equation (7.47) the numerical flux is reduced to

$$
g^{e o}(u, v)=\frac{u(a+|a|)}{4}+\frac{v(a-|a|)}{4} .
$$

In this case, $q=|a| \frac{\Delta t}{\Delta x}$ and the scheme is stable as soon as

$$
\Delta t \leq \frac{\Delta x^{2}}{\Delta x|a|+2 \nu} .
$$

In the nonlinear case, the numerical flux associated to the Engquist-Osher scheme is given by

$$
g^{e o}(u, v)=\frac{u(u+|u|)}{4}+\frac{v(v-|v|)}{4} .
$$

Generally speaking, the stability of these schemes for the nonlinear viscous Burgers equation can be obtained from the stability analysis for general 
conservative schemes, since they can be written in conservative form with the modified flux (7.46).

It is well known that such schemes admit the following viscous form:

$u_{j}^{n+1}=u_{j}^{n}-\frac{\Delta t}{\Delta x} \frac{f\left(u_{j+1}^{n}\right)-f\left(u_{j}^{n}\right)}{2}+\frac{Q_{j+1 / 2}\left(v_{j+1}-v_{j}\right)-Q_{j-1 / 2}\left(v_{j}-v_{j-1}\right)}{2}$,

where

$$
Q_{j+1 / 2}=\frac{\Delta t}{\Delta x}\left(f\left(u_{j+1}^{n}\right)+f\left(u_{j}^{n}\right)-2 g_{v i s}^{e o}\left(u_{j}^{n}, u_{j+1}^{n}\right)\right),
$$

and that the scheme is total variation diminishing (TVD) if (see [GoRa91], p. 136)

$$
\frac{\Delta t}{\Delta x}\left|\frac{f\left(u_{j+1}^{n}\right)+f\left(u_{j}^{n}\right)}{u_{j+1}^{n}-u_{j}^{n}}\right| \leq Q_{j+1 / 2} \leq 1 .
$$

Thus, this numerical scheme is stable if the following condition is satisfied:

$$
\left(\max _{j}\left|u_{j}^{0}\right|\right)^{2} \frac{\Delta t}{\Delta x}+2 \nu \frac{\Delta t}{\Delta x^{2}} \leq 1 .
$$

\subsubsection{Numerical Experiments for the Optimization Problem}

In this section we present some numerical experiments to illustrate the results of the previous sections. We pay special attention to showing the applicability of the alternating descent method.

We emphasize that the solutions obtained with each method may correspond to global minima or local ones since the gradient algorithm does not distinguish them.

We consider an exact solution of the Burgers equation obtained as a traveling wave

$$
u(x, t)=\frac{1}{2}\left(1-\tanh \frac{x-t / 2}{4 \nu}\right) .
$$

This solution is smooth for $\nu>0$ but, as $\nu \rightarrow 0$, it approaches a piecewise constant function with a discontinuity at $x=t / 2, t \in[0,1]$. We choose the final time $T=1$ and the target $u^{d}$, different for each value of the viscosity parameter, given by

$$
u^{d}=\frac{1}{2}\left(1-\tanh \frac{x-T / 2}{4 \nu}\right) .
$$

Note that the functional attains its minimum value, $J=0$, and a minimizer is given by

$$
u^{0, \min }=\frac{1}{2}\left(1-\tanh \frac{x}{4 \nu}\right) .
$$

The interval $(-6,6)$ has been chosen as the computational domain, and we have taken as boundary conditions, at each time step $t=t^{n}$, the value of the known target at the boundary. 
To illustrate the efficiency of the alternating descent method, we have solved the optimization problem with a descent method using the usual adjoint formulation and with the alternating descent method, for different values of the viscosity parameter $\nu=0.5,0.1$ and $\nu=0.01$.

We also consider $\Delta x=0.02$ and $\Delta t=\Delta x^{2} / 2$, which satisfies the stability condition (7.49).

It is interesting to compare the relation between the physical viscosity parameter $\nu$ and the numerical viscosity introduced by the Engquist-Osher scheme itself. Observe that the last term in (7.48) can be written as

$$
\left(|a| \frac{\Delta x}{2}+\nu\right) \frac{\Delta t}{\Delta x^{2}}\left(u_{j+1}^{n}-2 u_{j}^{n}+u_{j-1}^{n}\right),
$$

which allows us to compare the influence of these two quantities on the numerical solution. In the case $\nu=0.01$ and when $|a|=1$, the physical viscosity is of the order of the numerical viscosity introduced by the Engquist-Osher scheme for the inviscid Burgers equation, i.e., $|a| \frac{\Delta x}{2}=0.01|a|=\nu$. Thus, $\nu=0.01$ can be interpreted as the numerical solution of the inviscid Burgers equation.

Note that this is not an unusual situation in transonic aerodynamic applications of fluid dynamics problems. In those problems, the thickness of the shock wave is too small to be resolved by a computational mesh. The numerical dissipation dominates the physical one, unless an exceptionally fine mesh is set up. In these cases, it is natural to obtain approximate solutions using numerical methods for inviscid flows (see [Hi88], Chapter 22).

We solve the optimization problem starting either with $u^{0} \equiv 0$ or the following:

$$
u^{0}=\left\{\begin{array}{l}
2 \text { if } x<1 / 4 \\
0 \text { if } x \geq 1 / 4
\end{array}\right.
$$

which has a discontinuity at $x=1 / 4$. A discontinuous function is suitable for the alternating method while, for the classical adjoint method, a smooth initialization is a priori more natural.

In Figure 7.4 we show numerical experiments for three different values of the viscosity parameters $\nu=0.5,0.1$ and $\nu=0.01$ in different rows. At each row, the left figure corresponds to the initial data $u^{0}$ obtained after optimization when the gradient is computed with the adjoint method, initialized with $u^{0} \equiv 0$ and the $u^{0}$ given in (7.52), as well as the alternating method initialized with the discontinuous function in (7.52). In the figure on the right, the solutions at the final time $t=1$ are drawn.

In Figure 7.5 the values of the functional versus the iteration are shown for each method and the different values of $\nu$ described before.

We see that for large values of the viscosity $\nu$ the classical adjoint method starting with the smooth data $u^{0} \equiv 0$ is preferable. When $\nu$ becomes smaller, the efficiency of the algorithm does not depend very much on the initialization, and both $u^{0} \equiv 0$ and the one in (7.52) provide similar results. 

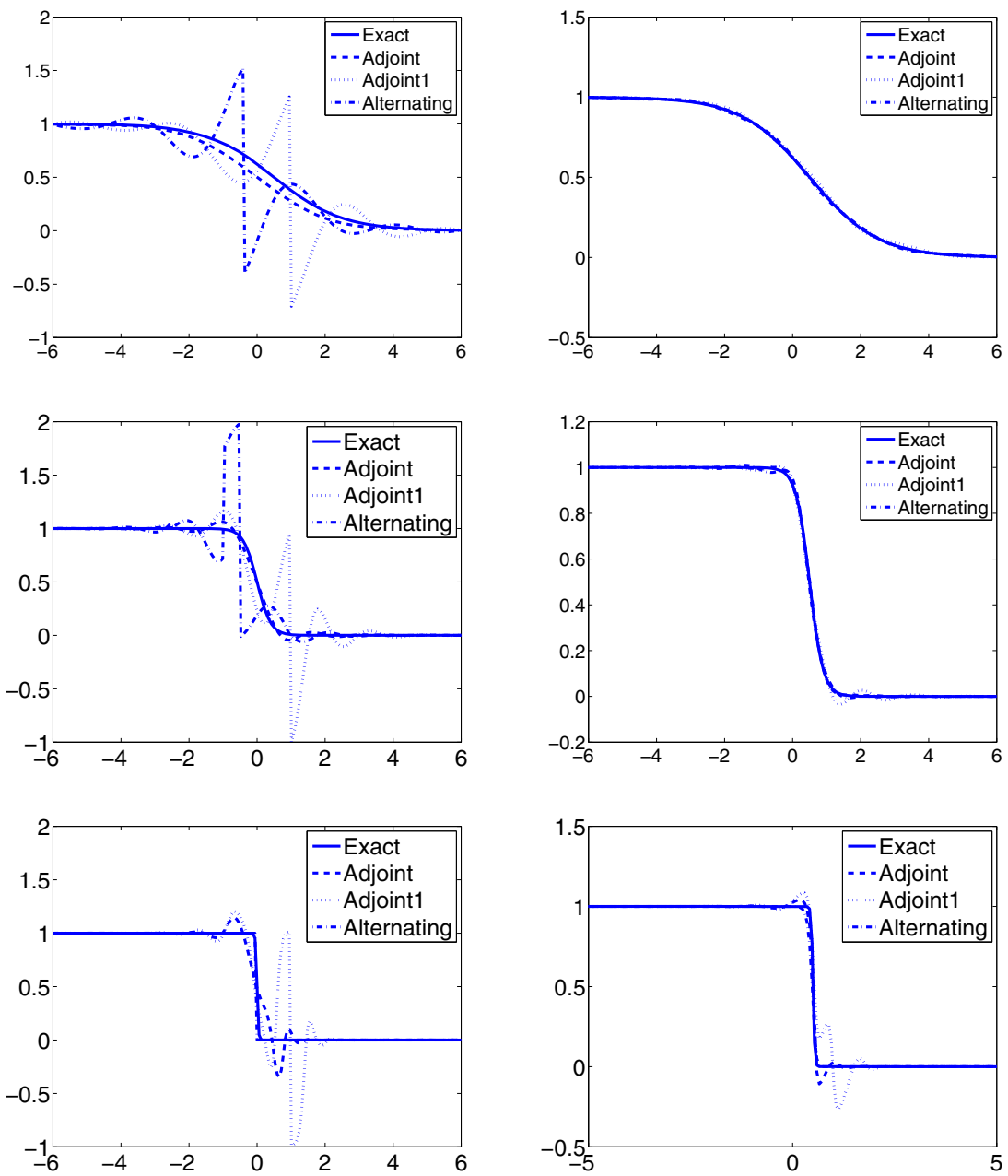

Fig. 7.4. The upper figures correspond to the value of the viscosity $\nu=0.5$, the middle ones correspond to $\nu=0.1$ and the lower ones correspond to $\nu=0.01$. The left figure of each row contains: Exact initial data (Exact), initial data obtained from the descent algorithm with the classical adjoint method initialized with $u^{0}=0$ (Adjoint), the same initialized with (7.52) (Adjoint1) and the alternating descent method described in this paper and initialized with (7.52) (Alternating). The right figure of each row contains: Exact solution at $t=1$ (Exact) and solutions obtained with the different methods described. 

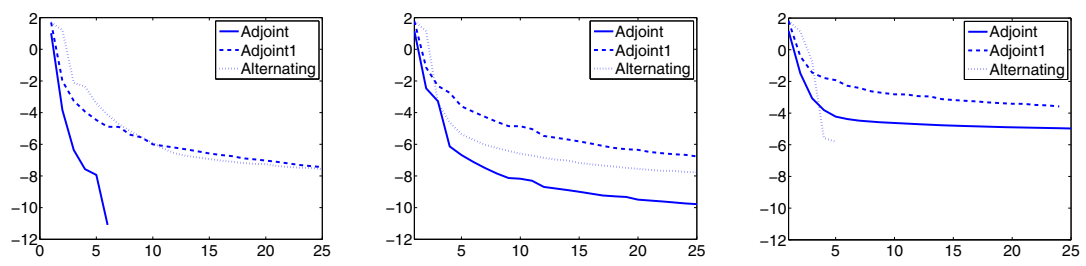

Fig. 7.5. Values of the functional versus iterations of the descent method, for the different methods with viscosities $\nu=0.5$ (left), $\nu=0.1$ (middle), and $\nu=0.01$ (right).
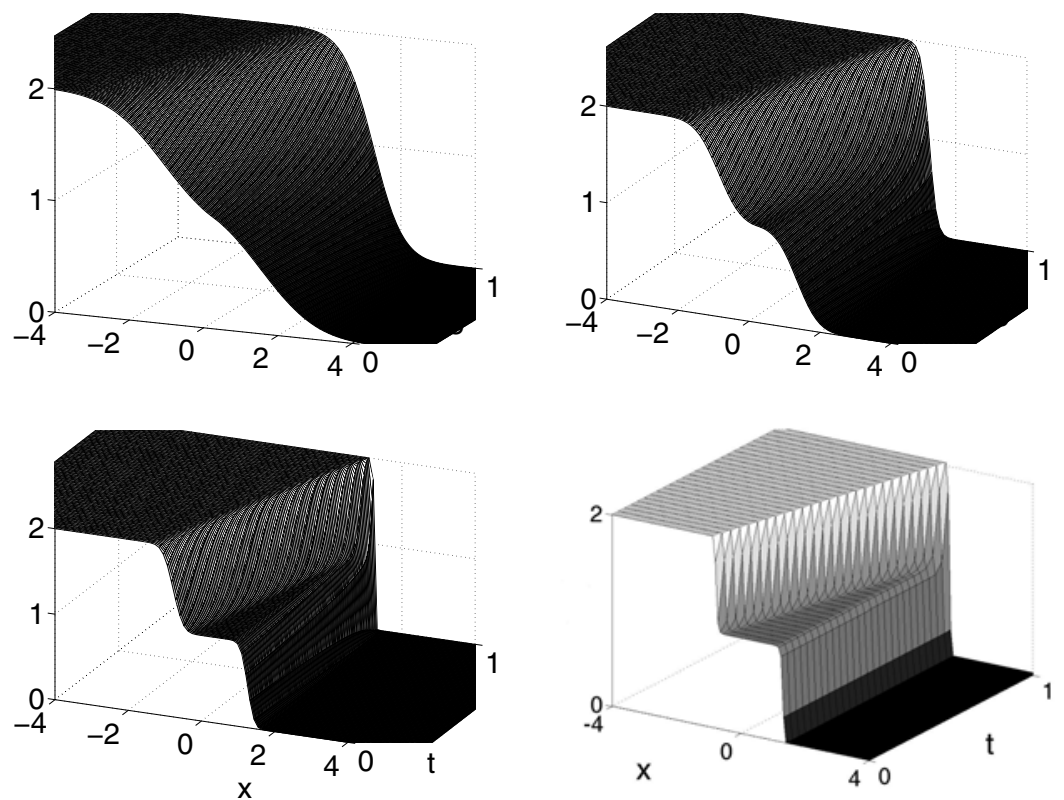

Fig. 7.6. Adjoint solutions corresponding to the solution $u$ in Figure 7.3 for different viscous values $\nu=0.5$ (upper left), $\nu=0.1$ (upper right), and $\nu=0.01$ (lower left) and the exact adjoint solution (lower right).

On the other hand, the alternating descent method is more efficient when the viscosity becomes sufficiently small, especially in those cases where $\nu$ is of the order of the numerical dissipation. Let us briefly explain this. In this nonlinear situation, the numerical dissipation is given by $|u| \frac{\Delta x}{2}$. Taking into account that our target is a function which takes values in the interval $[0,1]$, it is natural to assume that the numerical optimal solution will take values in a neighborhood of $[0,1]$. Thus, according to our choice of $\Delta x=0.02$, the 
numerical dissipation will be at most of the order of 0.01 , depending on the value of the numerical solution $u_{j}^{n}$ at each point of the mesh. If we incorporate a physical viscosity $\nu=0.01$, we introduce a perturbation which is of the order of the maximum value of the numerical dissipation.

In Figure 7.6 it is shown that, in this case, the solutions of the adjoint system are closer to the solutions of the adjoint system for the inviscid equation given in Figure 7.3.

Acknowledgement. This work is supported by Grant MTM2008-03541 of the MEC (Spain) and the SIMUMAT project of the CAM (Spain).

\section{References}

[BaPi02] Bardos, C., Pironneau, O.: A formalism for the differentiation of conservation laws. C.R. Acad. Sci. Paris Sér I, 335, 839-845 (2002).

[BaPi03] Bardos, C., Pironneau, O.: Derivatives and control in presence of shocks. J. Comput. Fluid Dynamics, 11, 383-392 (2003).

[BoJa98] Bouchut, F., James, F.: One-dimensional transport equations with discontinuous coefficients. Nonlinear Anal. Theory Appl., 32, 891-933 (1998).

[BoJa99] Bouchut, F., James, F.: Differentiability with respect to initial data for a scalar conservation law, in Proceedings Seventh Internat. Conf. on Hyperbolic Problems, Birkhäuser, Basel (1999), 113-118.

[BoJa05] Bouchut, F., James, F., Mancini, S.: Uniqueness and weak stability for multi-dimensional transport equations with one-sided Lipschitz coefficient. Ann. Sc. Norm. Super. Pisa Cl. Sci., 4, 1-25 (2005).

[BrOs88] Brenier, Y., Osher, S.: The discrete one-sided Lipschitz condition for convex scalar conservation laws. SIAM J. Numer. Anal., 25, 8-23 (1988).

[BrMa95a] Bressan, A., Marson, A.: A variational calculus for discontinuous solutions of systems of conservation laws. Comm. Partial Diff. Equations, 20, 1491-1552 (1995).

[BrMa95b] Bressan, A., Marson, A.: A maximum principle for optimally controlled systems of conservation laws. Rend. Sem. Mat. Univ. Padova, 94, 79-94 (1995).

[CaPa08] Castro, C., Palacios, F., Zuazua, E.: An alternating descent method for the optimal control of the inviscid Burgers equation in the presence of shocks. Math. Models Methods Appl. Sci., 18, 369-416 (2008).

[CaZu08] Castro, C., Zuazua, E.: On the flux identification problem for scalar conservation laws in the presence of shocks (preprint, 2008).

[DaLe95] Dal Maso, G., Le Floch, P., Murat, F.: Definition and weak stability of nonconservative products. J. Math. Pures Appl., 74, 458-483 (1995).

[EsVa93] Escobedo, M., Vázquez, J.L., Zuazua, E.: Asymptotic behavior and source-type solutions for a diffusion-convection equation. Arch. Rational Mech. Anal., 124, 43-65 (1993). 
[GaGu01] Garreau, S., Guillaume, P., Masmoudi, M.: The topological asymptotic for PDE systems: the elasticity case. SIAM J. Control Optim., 39, 17561778 (2001).

[GiPi01] Giles, M.B., Pierce, N.A.: Analytic adjoint solutions for the quasi-onedimensional Euler equations. J. Fluid Mech., 426, 327-345 (2001).

[Gl03] Glowinski, R.: Numerical Methods for Fluids. Part 3, Handbook of Numerical Analysis IX, Ciarlet, P., Lions, J.-L., eds., Elsevier, Amsterdam (2003).

[GoRa99] Godlewski, E., Raviart, P.A.: The linearized stability of solutions of nonlinear hyperbolic systems of conservation laws. A general numerical approach. Math. Comp. Simulations, 50, 77-95 (1999).

[GoRa91] Godlewski, E., Raviart, P.A.: Hyperbolic Systems of Conservation Laws, Ellipses, Paris (1991).

[GoOl98] Godlewski, E., Olazabal, M., Raviart, P.A.: On the linearization of hyperbolic systems of conservation laws. Application to stability, in Équations Différentielles et Applications, Gauthier-Villars, Paris (1998), 549-570.

[GoRa96] Godlewski, E., Raviart, P.A.: Numerical Approximation of Hyperbolic Systems of Conservation Laws, Springer, Berlin (1996).

[GoJa00] Gosse, L., James, F.: Numerical approximations of one-dimensional linear conservation equations with discontinuous coefficients. Math. Comput., 69, 987-1015 (2000).

[Hi88] Hirsch, C.: Numerical Computation of Internal and External Flows. Vols. 1 and 2, Wiley, New York (1988).

[JaSe99] James, F., Sepúlveda, M.: Convergence results for the flux identification in a scalar conservation law. SIAM J. Control Optim., 37, 869-891 (1999).

[Le02] LeVeque, R.: Finite Volume Methods for Hyperbolic Problems, Cambridge University Press, London (2002).

[Ma83] Majda, A.: The Stability of Multidimensional Shock Fronts, American Mathematical Society, Providence, RI (1983).

[Me03] Métivier, G.: Stability of multidimensional shocks. Course notes, http://www.math.u-bordeaux.fr/ metivier/cours.html (2003)

[MoPi04] Mohammadi, B., Pironneau, O.: Shape optimization in fluid mechanics. Annual Rev. Fluids Mech., 36, 255-279 (2004).

[NaJa00] Nadarajah, S., Jameson, A.: A comparison of the continuous and discrete adjoint approach to automatic aerodynamic optimization. AIAA Paper 2000-0667, 38th Aerospace Sciences Meeting and Exhibit, January 2000, Reno, NV.

[Ol57] Oleinik, O.: Discontinuous solutions of nonlinear differential equations. Amer. Math. Soc. Transl., 26, 95-172 (1957).

[U103] Ulbrich, S.: Adjoint-based derivative computations for the optimal control of discontinuous solutions of hyperbolic conservation laws. Systems Control Lett., 48, 313-328 (2003).

[Wh74] Whitham, G.B.: Linear and Nonlinear Waves, Wiley, New York (1974). 